\title{
Electronic structure of sub-10 $\mathrm{nm}$ colloidal silica nanoparticles measured by in situ photoelectron spectroscopy at the aqueous-solid interface
}

\author{
Matthew A. Brown, ${ }^{* a}$ Robert Seidel, ${ }^{b}$ Stephan Thürmer, ${ }^{b}$ Manfred Faubel, ${ }^{c}$ \\ John C. Hemminger, ${ }^{d}$ Jeroen A. van Bokhoven, ${ }^{a e}$ Bernd Winter $^{b}$ and Martin Sterrer $* f$ \\ Received 11th April 2011, Accepted 7th June 2011 \\ DOI: $10.1039 / \mathrm{clcp} 21131 \mathrm{e}$
}

X-Ray photoelectron spectroscopy has been extended to colloidal nanoparticles in aqueous solution using a liquid microjet in combination with synchrotron radiation, which allowed for depth-dependent measurements. Two distinct electronic structures are evident in the $\mathrm{Si} \mathbf{2 p}$ photoelectron spectrum of $7 \mathbf{n m}$ $\mathrm{SiO}_{2}$-nanoparticles at $\mathrm{pH}$ 10. A core-shell model is proposed where only the outermost layer of $\mathrm{SiO}_{2}$ nanoparticles, which is mainly composed of deprotonated silanol groups, $>\mathrm{Si}-\mathrm{O}^{-}$, interacts with the solution. The core of the nanoparticles is not affected by the solvation process and retains the same electronic structure as measured in vacuum. Future opportunities of this new experiment are also highlighted.

\section{Introduction}

Photoelectron spectroscopy (PES) is a powerful quantitative tool that can provide chemical specific insight into electronic and geometric structures at interfaces with depth-resolution. ${ }^{1}$ The main drawback of PES, however, is that it requires a vacuum environment and its application has therefore been limited to vacuum compatible systems that often do not represent the structural and chemical complexity of the real systems they strive to mimic.

In the attempt to appeal to a broader research community and to address scientific concerns arising from the pressure and material gaps which vacuum environments manifest, PES has recently been extended to ambient-gas ${ }^{2}$ and aqueous ${ }^{3,4}$ environments. These studies have proven valuable in fields as diverse as atmospheric chemistry, ${ }^{5-9}$ heterogeneous catalysis, ${ }^{10-13}$ and biological sciences. ${ }^{14,15}$ However, the extension of PES to the important class of colloidal nanoparticle (NP) systems has to date not been reported. Here we extend PES to the solid-liquid

${ }^{a}$ Institute for Chemical and Bioengineering, ETH Zurich, HCI G101, CH-8093 Zurich,Switzerland.E-mail: matthew.brown@chem.ethz.ch

${ }^{b}$ Helmholtz-Zentrum Berlin fur Materialien und Energie,

D-12489 Berlin, Germany

${ }^{c}$ Max-Planck-Institut fur Dynamik und Selbstorganisation,

D-37073 Gottingen, Germany

${ }^{d}$ Department of Chemistry, University of California Irvine, Irvine, 92697, USA

${ }^{e}$ Paul Scherrer Institute, CH-5232 Villigen, Switzerland

${ }^{f}$ Department of Chemical Physics, Fritz-Haber-Institut der

Max-Planck-Gesellschaft, Faradayweg 4-6, D-14195 Berlin,

Germany.E-mail:sterrer@fhi-berlin.mpg.de interface of NPs in aqueous solution (Fig. 1) and present the results of electronic structure measurements from aqueous colloidal silica NPs $\left(\mathrm{SiO}_{2}-\mathrm{NP}_{(\mathrm{aq})}\right)$ stabilized by $\mathrm{Na}_{2} \mathrm{O}$ at $\mathrm{pH} 10.0$ using energy-dependent X-ray photoelectron spectroscopy (XPS) from a free-flowing, equilibrated liquid jet. ${ }^{3,4}$

Colloidal nanoparticles have wide ranging applications in chemical, physical, material and biological sciences that extend beyond fundamental interest and into our everyday lives. For instance, in materials science colloidal NPs have found application as the emitters for thin film light-emitting diodes (LEDs), ${ }^{16}$ while $20 \mathrm{wt} \%$ aqueous colloidal silica is used as an additive for improving physiochemical and in vitro biological properties of calcium sulfate based nanocomposite bone cement. ${ }^{17}$ Several recent reviews provide a comprehensive list of the different applications of colloidal NPs. ${ }^{18,19}$

While there are many current applications for colloidal NPs, our microscopic understanding of their physical and electronic properties is far from complete. Broadening our basic atomicscale understanding of aqueous colloidal NPs requires interdisciplinary studies that bridge the different expertise of the chemical community. Here we show how PES can be used to gain valuable information regarding the detailed electronic

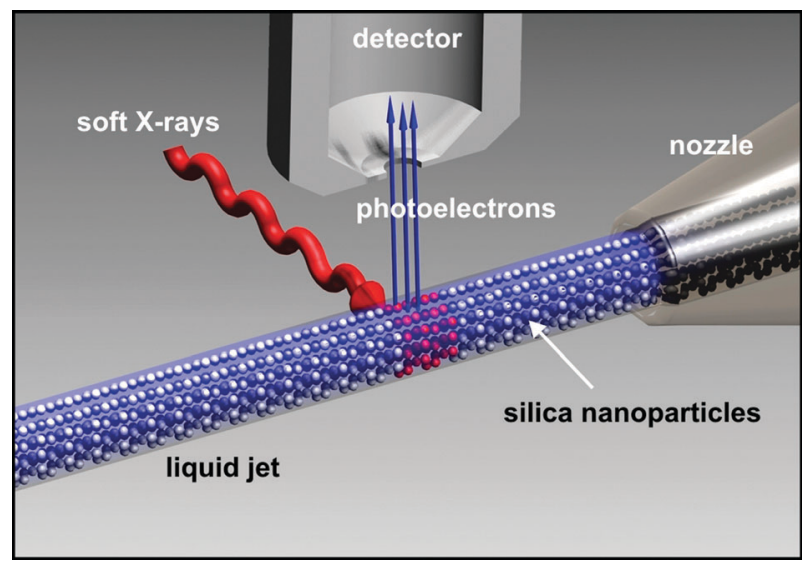

Fig. 1 The experimental setup depicting the extension of in situ PES to the solid-liquid interface of aqueous colloidal $\mathrm{SiO}_{2}$-NPs. The $24 \mu \mathrm{m}$ liquid jet is injected into vacuum where it spatially overlaps with the micro focus of the soft X-rays at the PGM-U41 beamline of the synchrotron radiation facility BESSY. Photoelectrons are collected using a differentially pumped hemispherical energy analyzer. 
structure of $\mathrm{SiO}_{2}-\mathrm{NP}_{(\mathrm{aq})}$ in their native aqueous state. To our knowledge the spectra presented here are the first energydependent electronic structure measurements of chargestabilized colloidal NPs in aqueous solution.

\section{Experimental}

Our X-ray PES measurements used a $24 \mu \mathrm{m}$ aqueous liquid jet (MicroLiquids $\mathrm{GmbH}$ ) of a LUDOX SM-30 colloidal silica dispersion (Sigma-Aldrich, 7-nm particle diameter, spherical, amorphous, non-porous, surface area $=345 \mathrm{~m}^{2} / \mathrm{g}$ ) diluted to $20 \mathrm{wt} \%$ and injected into the measurement chamber of the LiquidJetPES endstation ${ }^{3,4}$ at the PGM-U41 beamline of the synchrotron radiation facility BESSY. The liquid jet was operated at a flow of $0.65 \mathrm{ml} / \mathrm{min}$ and at $279 \mathrm{~K}$ in a vacuum of $1 \times 10^{-4} \mathrm{mbar}$. Using variable energy incident soft X-rays the $\mathrm{Si} 2 \mathrm{p}$ region was collected at photoelectron kinetic energies (eKEs) of 100, 400 and $700 \mathrm{eV}$. Carrying out experiments as a function of eKE allows for a depth profile into solution based on the photoelectron's inelastic mean free path (IMFP). ${ }^{3}$ By increasing the eKE of the experiment (which is achieved here by tuning the incident photon energy) the relative contribution of photoelectrons generated further below the liquid-vapor interface can also be increased. The $\mathrm{O}$ 1s spectral region was collected as well but no features could be distinguished above the strong signal intensity of the water. Reported binding energies are relative to the vacuum level and calibrated by the $1 b_{1}$ orbital of liquid water. ${ }^{21}$

For the calculation presented in Fig. 3 IMFPs of bulk silica and of water were used to determine the core-shell thickness of the NP. ${ }^{20}$ The photoelectrons were subject to two exponentially weighted damping factors, the first for the $\mathrm{SiO}_{2} \mathrm{NP}$ and the second for water (discussed in more detail in the following

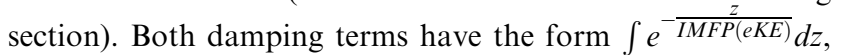
where $z$ is first the distance into the NP, below the surface, where the PE is created (accounts for inelastic scattering as the PE exits the NP) and then the distance through the water that the PE must travel before escaping into vacuum (accounts for inelastic scattering as the PE travels through the liquid media).

\section{Results and discussion}

Fig. 2 shows the PE spectra of the $\mathrm{Si} 2 \mathrm{p}$ region for the $20 \mathrm{wt} \%$ colloidal suspension of $\mathrm{SiO}_{2}-\mathrm{NPS}_{(\mathrm{aq})}$ at the three different probe depths (eKEs) of this study. All spectra are asymmetrical in line shape with a shoulder on the low BE side of the main peak that requires a second component to properly fit. The main peak in all spectra is centered at a binding energy (BE) of $107.8 \mathrm{eV}$ with a full-width-half-maximum (FWHM) of $1.5 \mathrm{eV}$. The lower BE peak in each spectrum is centered at $106.5 \mathrm{eV}$ and has a FWHM of $2.0 \mathrm{eV}$. The ratio of the integrated area of the high to low BE peaks increases from 4.9 at $100 \mathrm{eV}$ eKE to 6.3 at $400 \mathrm{eV}$ eKE, and 6.9 at $700 \mathrm{eV}$ eKE. Such an increase in ratio as the eKE is increased suggests that the low-BE peak originates from a surface component of the NP and the high-BE peak is from the bulk of the NP (here we refer to the interior of the NP). Our assignment of the peak centered at $107.8 \mathrm{eV}$ as bulk $\mathrm{SiO}_{2}-\mathrm{NP}_{(\mathrm{aq})}$ is in agreement with a previous study of extended solid films of $\mathrm{SiO}_{2}$ referenced to the vacuum level where a BE of $108( \pm 0.3)$ eV for Si $2 p$ was reported. ${ }^{22}$

Si $2 p$ BE values lower than the bulk $\mathrm{SiO}_{2} \mathrm{BE}$ are typically observed in UHV studies of partially oxidized $\mathrm{Si}$ films and are assigned to under-coordinated $\mathrm{Si}$ sites of reduced oxidation state. ${ }^{23}$ Recently, PES measurements have also been reported for free silica nanoparticles in the gas phase, ${ }^{24,25}$ and neither of these studies reported an asymmetry, or two-component fit to the Si $2 p$ peak as seen in the present study. From the gas-phase PES studies, and under the belief that under-coordinated $\mathrm{Si}$ would not be present in aqueous solution, we infer that the two-component spectral structure arises from the solvation of the silica NPs in the aqueous $\mathrm{pH} 10$ solution. Based on the depth-resolved intensity ratio of the spectra in Fig. 2, and the idea that the two-component structure of the $\mathrm{Si} 2 \mathrm{p}$ region is a direct result of NP solvation, the lower $\mathrm{BE}$ component is indeed assigned as the outermost surface of the $\mathrm{SiO}_{2}-\mathrm{NP}_{(\mathrm{aq})}$, or the shell of the NP, that directly interacts with the solution.

The photoelectron kinetic energy-dependent Si $2 p$ measurements shown in Fig. 2 allow for the shell thickness of the $\mathrm{SiO}_{2}-\mathrm{NP}_{(\mathrm{aq})}$ to be determined. We calculated the expected PES signal ratio from a solvated core-shell NP as a function of shell thickness assuming both the shell and the core have constant
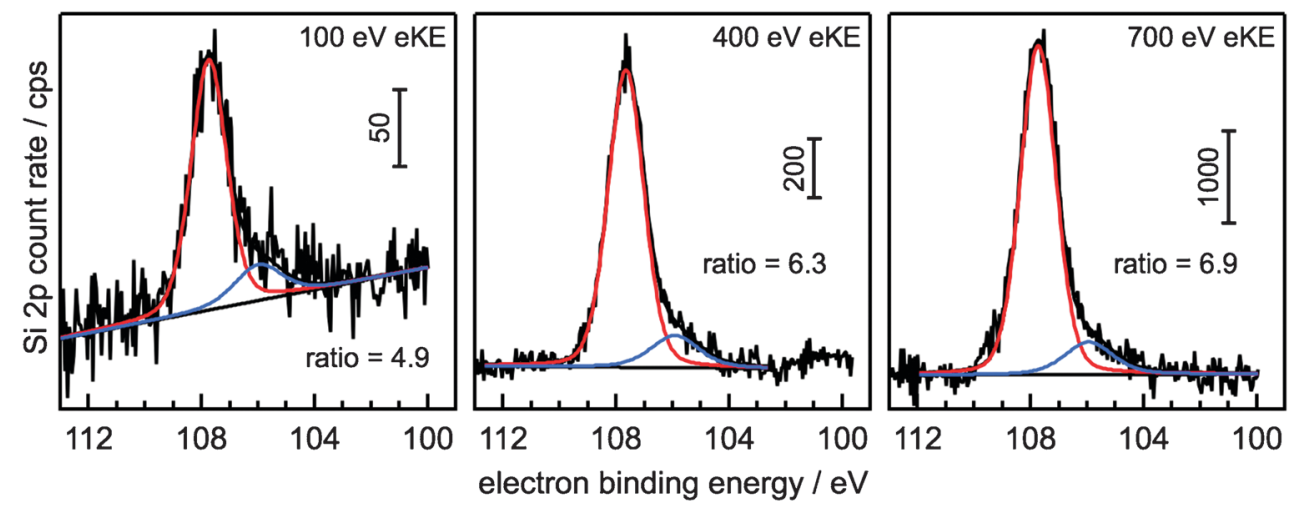

Fig. 2 Si 2 p photoelectron spectra of $20 \mathrm{wt} \% \mathrm{SiO}_{2}-\mathrm{NP}_{(\mathrm{aq})}$ at three different probe depths (eKEs). The binding energy scale is relative to the vacuum level. The asymmetry of the spectra requires a two-component fit as shown in red (high BE) and blue (low BE). The high BE component originates from the NP core whereas the low BE component can be assigned to the outermost surface layer of the NP. The ratio of the integrated peak area, high $\mathrm{BE}$ to low $\mathrm{BE}$, is shown. 

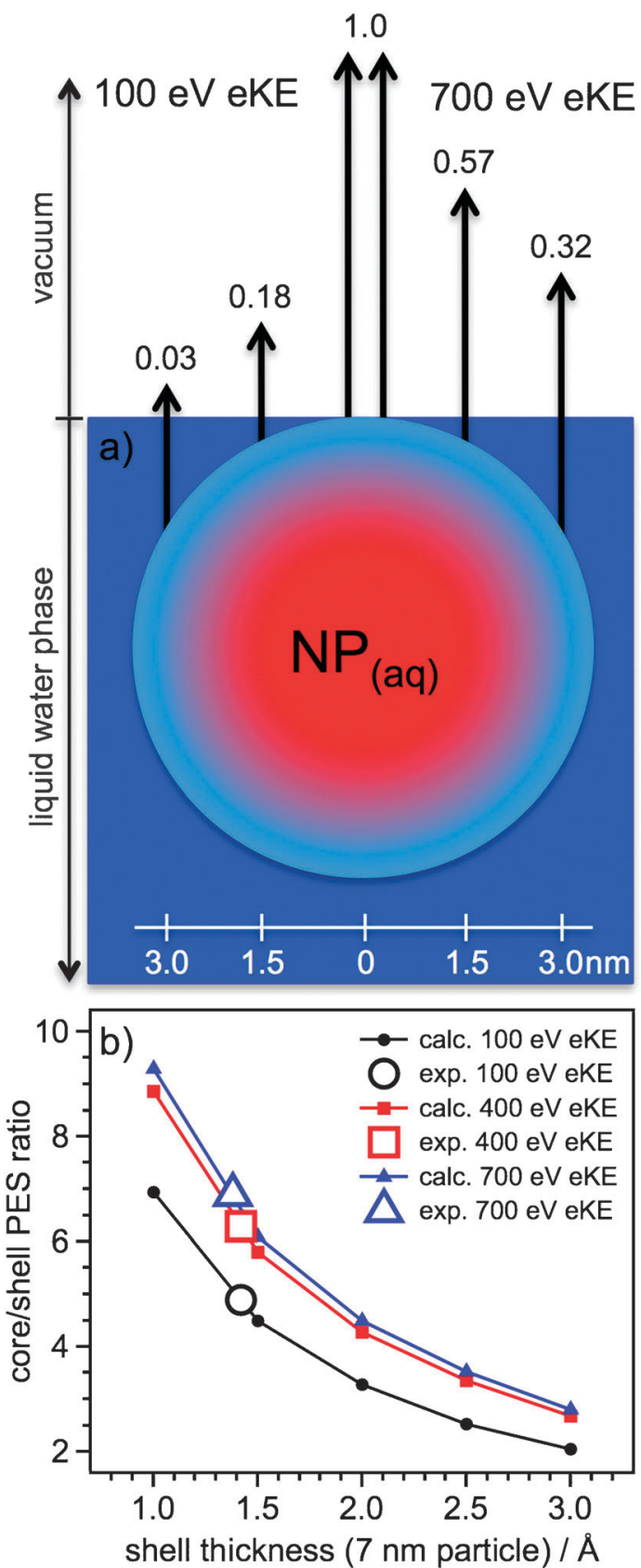

Fig. 3 (a) Weighted contribution to the overall signal intensity of a photoelectron generated at the surface of a 7-nm spherical nanoparticle located at the solution-vacuum interface for photoelectrons of $100 \mathrm{eV}$ and $700 \mathrm{eV}$ eKE. Photoelectrons generated on the surface of the nanoparticle, but below the liquid-vapor interface are weighted with an exponential damping due to scattering as they pass through the liquid phase and into vacuum. By comparison, the relative contribution of photoelectrons generated at the (top-half) surface of nanoparticles in gas phase or supported on substrates and measured in vacuum are all unity. (b) Predicted core/shell PES ratio of a solvated 7-nm nanoparticle as a function of shell thickness. The experimental data are also shown and are consistent, at all energies, with a shell thickness of $1.4 \AA$.

densities (Fig. 3b). The liquid solvent presents a unique case for such a calculation. In addition to the IMFP from within the NP, which is accounted for here by using the well-known
IMFP for $\mathrm{SiO}_{2},{ }^{20}$ a second attenuation from the water must also be considered once the PE has left the NP but before it escapes into vacuum. Fig. 3a highlights the effect of this second attenuation and shows the weighted contribution of a photoelectron generated at the surface of the solvated NP to the overall signal intensity as a function of depth into solution (below the liquid-vapor interface) at both $100 \mathrm{eV}$ eKE and $700 \mathrm{eV}$ eKE for a spherical 7-nm diameter NP that resides at the liquid-vapor interface. At $100 \mathrm{eV}$ eKE a photoelectron generated on the surface of the solvated NP, but at a depth $>3 \mathrm{~nm}$ below the liquid-vapor interface (see Fig. 3a), effectively contributes zero to the measured signal, whereas a photoelectron generated at the same position of the NP but with $700 \mathrm{eV}$ eKE, where the IMFP is $\sim 3 \times$ longer than at $100 \mathrm{eV} \mathrm{eKE},{ }^{20}$ contributes more noticeably to the observed signal intensity. The second damping due to the solvent is not encountered in gas phase PES measurements where inelastic collisions are only present within the material under investigation and therefore by contrast, in a gas phase PES experiment these two photoelectrons generated at the surface of the NP would have the same weighted contribution to the overall signal intensity (1.0). The increased contribution to the overall signal intensity of photoelectrons generated further below the liquid-vapor interface at $700 \mathrm{eV}$ eKE compared to $100 \mathrm{eV}$ eKE allows for a depth profile into solution. Fig. $3 \mathrm{~b}$ shows the results of our calculation at the three different eKEs of this study for shell thicknesses that range from 1.0-3.0 $\AA$. The results of the experiment, obtained by integrating the peak areas of the fits shown in Fig. 2 are shown in Fig. 3b overlaying the calculated values. The data, at all eKEs, is consistent with a shell thickness of $1.4 \AA$. The experimental results are consistent with only the outermost layer of the $\mathrm{SiO}_{2}-\mathrm{NP}_{(\mathrm{aq})}$ being affected by solvation. The electronic structure of the NP core is essentially unaffected as evidenced by a measured $\mathrm{BE}$ characteristic of bulk $\mathrm{SiO}_{2}$ measured in vacuum.

According to the $\mathrm{pH}$-dependent acid-base equilibria, at a solution $\mathrm{pH}$ of 10 the silica surface is mainly terminated by deprotonated silanol groups, $>\mathrm{Si}-\mathrm{O}^{-}$. In a previous XPS study, characteristic BE shifts were identified in the Si $2 p$ peak for the different surface species present on polycrystalline quartz samples after contact with solutions in the $\mathrm{pH}$ range $1-10$. A shift to $\sim 1.5 \mathrm{eV}$ lower $\mathrm{BE}$ compared to bulk $\mathrm{SiO}_{2}$ was observed at high $\mathrm{pH}$ values arising from the presence of $>\mathrm{Si}-\mathrm{O}^{-}$groups. $^{26}$ This observation is consistent with our in situ measurement and corroborates the assignment of the low BE component of the $\mathrm{Si} 2 \mathrm{p}$ spectra to deprotonated silanol groups present at the surface of the NPs in an aqueous solution of $\mathrm{pH} 10$. We attribute the increased FWHM of the low BE Si 2 p peak (2.0 eV compared with 1.5 in the NP bulk; Fig. 2) to a larger distribution of solvation configurations on the surface of the NP in solution than in the bulk. Specifically, heterogeneity in the surface structure, hydrogen bonding between $>\mathrm{Si}-\mathrm{O}^{-}$and other surface species which may be present, such as neutral silanol groups $(>\mathrm{Si}-\mathrm{OH})$ and adsorbed molecular water, as well as adsorbed counterions $\left(\mathrm{Na}^{+}\right)$may all be present at the surface of the NP in solution. Overall, these different configurations result in the observed broadening of the surface Si 2 p peak, however, the exact assignment remains unresolved in the current study. 


\section{Conclusions}

We have extended liquid based PES, originally developed by Siegbahn and co-workers for atomic and molecular solutes in non volatile solution, ${ }^{27,28}$ to the solid-liquid interface of aqueous NP using a liquid microjet in combination with synchrotron radiation. Our measurements identified a distinct electronic structure in the $\mathrm{Si} 2 \mathrm{p}$ spectrum of $7 \mathrm{~nm}$ colloidal $\mathrm{SiO}_{2}$ nanoparticles that can be assigned to deprotonated silanol groups, $>\mathrm{Si}-\mathrm{O}^{-}$, on the surface of the nanoparticles. Based on a core-shell type model where the NP resides at the liquid-vapor interface, the surface layer is $1.4 \AA$ thick. The solvation process affects only the outermost layer of the nanoparticle while the core of the NP retains the electronic structure of bulk $\mathrm{SiO}_{2}$.

The extension of PES to aqueous colloidal nanoparticles (solid-liquid interface) is a development that now affords further research opportunity. One could imagine this becoming a standard research tool in the colloidal science community to establish, e.g., electronic structure of aqueous colloidal systems during the nucleation and growth stages of synthesis and to understand electronic changes that accompany charge transfer processes at the interface of organic coated aqueous nanoparticles. Further extending the technique to use hard $\mathrm{X}$-rays would allow for increased probing depth into solution and for detailed spatial distributions of the nanoparticles to be determined.

\section{Acknowledgements}

M.A.B and M.S. are indebted to Hajo Freund for his continued support. This work was supported by the AvH Foundation and ETH Zurich through postdoctoral fellowships (M.A.B.) and the DFG (Project WI 1327/3-1) (B.W.).

\section{Notes and references}

1 D. Briggs and M. P. Seah, Practical Surface Analysis, Auger and $X$-ray Photoelectron Spectroscopy, John Wiley and Sons, Chichester, 2nd edn, 1983, vol. 1.

2 H. Bluhm, J. Electron Spectrosc. Relat. Phenom., 2010, 177, 71.

3 M. A. Brown, M. Faubel and B. Winter, Annu. Rep. Prog. Chem., Sect. C, 2009, 105, 174.

4 B. Winter and M. Faubel, Chem. Rev., 2006, 106, 1176.

5 S. Ghosal, J. C. Hemminger, H. Bluhm, B. S. Mun, E. L. D. Hebenstreit, G. Ketteler, D. F. Ogletree, F. G. Requejo and M. Salmeron, Science, 2005, 307, 563.
6 M. A. Brown, B. Winter, M. Faubel and J. C. Hemminger, J. Am. Chem. Soc., 2009, 131, 8354.

7 S. Ghosal, M. A. Brown, H. Bluhm, M. J. Krisch, M. Salmeron, P. Jungwirth and J. C. Hemminger, J. Phys. Chem. A, 2008, 112, 12378.

8 M. J. Krisch, R. D'Auria, M. A. Brown, D. J. Tobias, J. C. Hemminger, M. Ammann, D. E. Starr and H. Bluhm, J. Phys. Chem. C, 2007, 111, 13497.

9 M. A. Brown, R. D’Auria, I. F. W. Kuo, M. J. Krisch, D. E. Starr, H. Bluhm, D. J. Tobias and J. C. Hemminger, Phys. Chem. Chem. Phys., 2008, 10, 4778.

10 F. Tao, M. E. Grass, Y. W. Zhang, D. R. Butcher, J. R. Renzas, Z. Liu, J. Y. Chung, B. S. Mun, M. Salmeron and G. A. Somorjai, Science, 2008, 322, 932.

$11 \mathrm{H}$. Bluhm, M. Havecker, A. Knop-Gericke, E. Kleimenov, R. Schlogl, D. Teschner, V. I. Bukhtiyarov, D. F. Ogletree and M. Salmeron, J. Phys. Chem. B, 2004, 108, 14340.

12 M. Havecker, R. W. Mayer, A. Knop-Gericke, H. Bluhm, E. Kleimenov, A. Liskowski, D. Su, R. Follath, F. G. Requejo, D. F. Ogletree, M. Salmeron, J. A. Lopez-Sanchez, J. K. Bartley, G. J. Hutchings and R. Schlogl, J. Phys. Chem. B, $2003, \mathbf{1 0 7}, 4587$

13 H. Bluhm, M. Havecker, A. Knop-Gericke, M. Kiskinova, R. Schlogl and M. Salmeron, MRS Bull., 2007, 32, 1022.

14 D. Nolting, E. F. Aziz, N. Ottosson, M. Faubel, I. V. Hertel and B. Winter, J. Am. Chem. Soc., 2007, 129, 14068.

15 D. Nolting, N. Ottosson, M. Faubel, I. V. Hertel and B. Winter, J. Am. Chem. Soc., 2008, 130, 8150.

16 S. Coe-Sullivan, W.-K. Woo, J. S. Steckel, M. Bawendi and V. Bulovic, Org. Electron., 2003, 4, 123.

17 S. Borhan, S. Hesaraki and S. Ahmadzazeh-Asl, J. Mater. Sci.: Mater. Med., 2010, 21, 3171.

18 D. V. Talapin, J.-S. Lee, M. V. Kovalenko and E. V. Shevchenko, Chem. Rev., 2010, 110, 389.

19 T. Pellegrino, S. Kudera, T. Liedl, A. Muñoz Javier, L. Manna and W. J. Parak, Small, 2005, 1, 48.

20 C. J. Powell and A. Jablonski, NIST Electron Inelastic-Mean-Free-Path Database-Version 1.1, National Institute of Standards and Technology, Gaithersburg, MD, 2000.

21 B. Winter, R. Weber, W. Widdra, M. Dittmar, M. Faubel and I. V. Hertel, J. Phys. Chem. A, 2004, 108, 2625.

22 A. Bianconi, Surf. Sci., 1979, 89, 41.

23 F. J. Himpsel, F. R. McFeely, A. Taleb-Ibrahimi, J. A. Yarmoff and G. Hollinger, Phys. Rev. B, 1988, 38, 6084.

24 E. R. Mysak, D. E. Starr, K. R. Wilson and H. Bluhm, Rev. Sci. Instrum., 2010, 81, 016106.

25 J. Meinen, S. Khasminskaya, M. Eritt, T. Leisner, E. Antonsson, B. Langer and E. Rühl, Rev. Sci. Instrum., 2010, 81, 085107.

26 Y. Duval, J. A. Mielczarski, O. S. Pokrovsky, E. Mielczarski and J. J. Ehrhardt, J. Phys. Chem. B, 2002, 106, 2937.

27 H. Siegbahn, L. Asplund, P. Kelfve and K. Siegbahn, J. Electron Spectrosc. Relat. Phenom., 1975, 7, 411.

28 H. Siegbahn, S. Svensson and M. Lundholm, J. Electron Spectrosc. Relat. Phenom., 1981, 24, 205. 